\title{
Interactions of Charmed Mesons with Light Pseudoscalar Mesons from Lattice QCD and Implications on the Nature of the $D_{s 0}^{*}(2317)$
}

\author{
Liuming Liu* ${ }^{* a}$, Kostas Orginos ${ }^{b, c}$, Feng-Kun Guo ${ }^{a}$, Christoph Hanhart ${ }^{d}$, and \\ UIf-G. Meißner ${ }^{a, d}$ \\ ${ }^{a}$ Helmholz-Institut für Strahlen- und Kernphysik and Bethe Center for Theoretical Physics, \\ Universität Bonn, D-53115 Bonn, Germany \\ ${ }^{b}$ Department of Physics, College of William and Mary, Williamsburg, VA 23187-8795, USA \\ ${ }^{c}$ Thomas Jefferson National Accelerator Facility, Newport News, VA 23606, USA \\ ${ }^{d}$ Institute for Advanced Simulation, Institut für Kernphysik and Jülich Center for Hadron Physics, \\ Forschungszentrum Jülich, D-52425 Jülich, Germany \\ E-mail: liuming@hiskp.uni-bonn.de, kostas@wm.edu, \\ fkguo@hiskp.uni-bonn.de, c.hanhartefz-juelich.de, \\ meissnerehiskp.uni-bonn.de
}

We study the scattering of light pseudoscalar mesons $(\pi, K)$ off charmed mesons $\left(D, D_{S}\right)$ in full lattice QCD. The $S$-wave scattering lengths are calculated using Lüscher's finite volume technique. We use a relativistic formulation for the charm quark. For the light quark, we use domainwall fermions in the valence sector and improved Kogut-Susskind sea quarks. We calculate the scattering lengths of isospin-3/2 $D \pi, D_{s} \pi, D_{s} K$, isospin-0 $D \bar{K}$ and isospin- $D \bar{K}$ channels on the lattice. For the chiral extrapolation, we use a chiral unitary approach to next-to-leading order, which at the same time allows us to give predictions for other channels. It turns out that our results support the interpretation of the $D_{s 0}^{*}(2317)$ as a $D K$ molecule. At the same time, we also update a prediction for the isospin breaking hadronic decay width $\Gamma\left(D_{s 0}^{*}(2317) \rightarrow D_{s} \pi\right)$ to $(133 \pm 22) \mathrm{keV}$.

31st International Symposium on Lattice Field Theory - LATTICE 2013

July 29 - August 3, 2013

Mainz, Germany

${ }^{*}$ Speaker. 


\section{Introduction}

In 2003, the BaBar Collaboration discovered a positive-parity scalar charm-strange meson $D_{s 0}^{*}(2317)$ with a very narrow width [1]. The state was confirmed later by the CLEO Collaboration [2]. The discovery of this state has inspired heated discussions in the past decade. The key point is to understand the low mass of this state, which is more than $100 \mathrm{MeV}$ lower than the prediction for the lowest scalar $c \bar{s}$ meson in, for instance, the Godfrey-Isgur quark model [3]. There are several interpretations of its structure, such as being a $D K$ molecule, the chiral partner of the pseudoscalar $D_{s}$, a conventional $c \bar{s}$ state, coupled-channel effects between the $c \bar{s}$ state and $D K$ continuum etc. In order to distinguish them, one has to explore the consequences of each interpretation, and identify quantities which have different values in different interpretations. Arguably the most promising quantity is the isospin breaking width $\Gamma\left(D_{s 0}^{*}(2317) \rightarrow D_{s} \pi\right)$. It is of order $10 \mathrm{keV}$ if the $D_{s 0}^{*}(2317)$ is a $c \bar{s}$ meson, while it is of order $100 \mathrm{keV}$ in the $D K$ molecular picture due to its large coupling to $D K$ and the proximity of the $D K$ threshold. Thus, the study of $D K$ interaction is very important in order to understand the structure of $D_{s 0}^{*}(2317)$. Although a direct simulation of the $D K(I=0)$ channel suffers from disconnected diagrams, one may obtain useful information on the $D K$ interaction by calculating the scattering lengths of the disconnected-diagram-free channels which can be related to $D K(I=0)$ through $\mathrm{SU}(3)$ flavor symmetry. This is the strategy we will follow in this paper.

In this work we employ the "coarse" $(a \simeq 0.125 \mathrm{fm})$ gauge configurations generated by the MILC Collaboration [4] using the one-loop tadpole-improved gauge action [5], where both $\mathscr{O}\left(a^{2}\right)$ and $\mathscr{O}\left(g^{2} a^{2}\right)$ errors are removed. For the fermions in the vacuum, the asqtad-improved KogutSusskind (staggered) action [6] is used. For the valence light quarks (up, down and strange) we use the five-dimensional Shamir domain-wall fermion action [7]. The domain-wall fermion action introduces a fifth dimension of extent $L_{5}$ and a mass parameter $M_{5}$; in our case, the values $L_{5}=16$ and $M_{5}=1.7$, both in lattice units, were chosen. For the charm quark we use a relativistic heavy quark action motivated by the Fermilab approach [8].

We use Lüscher's finite volume technique $[9,10]$ to calculate the scattering lengths. We then use unitarized chiral perturbation theory to extrapolate our results to the physical pion mass. Having fitted the appearing low-energy constants (LECs) to the lattice data, we are able to make predictions for other channels, in particular for the isospin zero, strangeness one channel in which the $D_{s 0}^{*}(2317)$ resides.

This work has been presented in Ref. [11]. For more details please see Ref. [11] and the references therein.

\section{Numerical Results}

We calculate the scattering lengths of the following five channels on lattice. The interpolating operators for these two-particle states are

$$
\mathscr{O}_{D_{s} \pi}=D_{s}^{-} \pi^{+}, \quad \mathscr{O}_{D \pi}^{I=3 / 2}=D^{+} \pi^{+}, \quad \mathscr{O}_{D_{s} K}=D_{s}^{+} K^{+}, \quad \mathscr{O}_{D \bar{K}}^{I=1}=D^{+} \bar{K}^{0}, \quad \mathscr{O}_{D \bar{K}}^{I=0}=D^{+} K^{-}-D^{0} \bar{K}^{0},
$$

where $D_{s}^{-}, D_{s}^{+}, D^{+}, K^{0}, K^{-}, K^{+}$and $\pi^{+}$are single particle operators which are of the form $\overline{q_{1}} \gamma_{5} q_{2}$. 
Lüscher has shown that the scattering phase shift is related to the energy shift $(\Delta E)$ in the total energy of two interacting hadrons in a finite box $[9,10]$. We use the formula [12]

$$
p \cot \delta(p)=\frac{1}{\pi L} \mathbf{S}\left(\left(\frac{p L}{2 \pi}\right)^{2}\right),
$$

where $p$ is the center-of-mass momentum, $\delta(p)$ is the $\mathrm{S}$-wave phase shift. The $\mathbf{S}$ function is defined as $\mathbf{S}(x)=\sum_{\mathbf{j}}^{|\mathbf{j}|<\Lambda} \frac{1}{\mid \mathbf{j}^{2}-x}-4 \pi \Lambda$.

In effective range approximation, the quantity $p \cot \delta(p)$ can be expanded as $p \cot \delta(p)=$ $\frac{1}{a}+\mathscr{O}\left(p^{2}\right)$ for $p^{2} \ll 1$. We ignore the terms that are higher order in $p^{2}$ and calculate the scattering length by

$$
a=\pi L / \mathbf{S}\left(\left(\frac{p L}{2 \pi}\right)^{2}\right) .
$$

To extract the energy shift $\triangle E$, we define a ratio $R^{h_{1} h_{2}}(t)=\frac{G^{h_{1} h_{2}(t)}}{G^{h_{1}}(t) G^{h_{2}}(t)} \longrightarrow \exp (-\triangle E \cdot t)$, where $G^{h_{1} h_{2}}(t)=\left\langle\mathscr{O}_{h_{1} h_{2}}(t)^{\dagger} \mathscr{O}_{h_{1} h_{2}}(0)\right\rangle, G^{h_{1}}(t, 0)$ and $G^{h_{2}}(t, 0)$ are two-point correlation functions. $\triangle E$ is obtained by fitting $R^{h_{1} h_{2}}(t)$ to a single exponential in the region where the effective mass exhibits a plateau.

For each channel, we calculate the ratio $R^{h_{1} h_{2}}$ at two different charm quark masses (denoted $m_{1}=0.2034$ and $m_{2}=0.2100$ ) and four different light valence quark masses corresponding to the four ensembles (m007, m010, m020 and m030) with pion masses approximately $301 \mathrm{MeV}, 364$ $\mathrm{MeV}, 511 \mathrm{MeV}$ and $617 \mathrm{MeV}$, respectively. Figure 1 shows the effective energy shifts of each channel calculated from ensemble m007 at the bare charm-quark mass $m_{2}=0.2100$. The fits of the energy shifts for other ensembles are similar. The energy shifts are linearly extrapolated to the physical charm-quark mass on each ensemble.
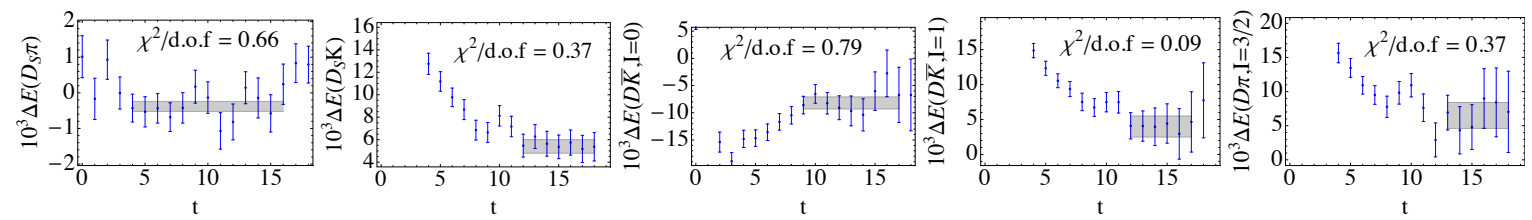

Figure 1: Effective energy shifts plots of the scattering channels $D_{s} \pi, D_{s} K, D \bar{K}(I=0), D \bar{K}(I=1)$, $D \pi(I=3 / 2)$. All plots are for ensemble m007. The grey bars show the fitted energy shifts and the fitting ranges. The height of the grey bars show the statistical errors.

\section{Chiral extrapolations of the scattering lengths}

Because the simulations are performed at unphysical quark masses, chiral extrapolation is necessary in order to obtain the values of scattering lengths at the physical quark masses. These scattering lengths were first calculated in Ref. [13] using a unitarized chiral approach. Here we take the same route as Ref. [13], and resum the chiral amplitude up to next-to-leading order, which is $\mathscr{O}\left(p^{2}\right)$. The resummed amplitude in the on-shell approximation reads

$$
T(s)=V(s)[1-G(s) V(s)]^{-1},
$$

where $V(s)$ is the $S$-wave projection of the $\mathscr{O}\left(p^{2}\right)$ scattering amplitude, and $G(s)$ is the scalar loop function regularized by a subtraction constant $\tilde{a}(\lambda)$. The details of $V(s)$ and $G(s)$ are given in 


\begin{tabular}{|c|cccccc|}
\hline Fitting range & $\chi^{2} /$ d.o.f & $\tilde{a}(\lambda=1 \mathrm{GeV})$ & $h_{24}$ & $h_{4}^{\prime}$ & $h_{35}$ & $h_{5}^{\prime}$ \\
\hline $\mathrm{m} 007-\mathrm{m} 020$ & 1.06 & $-1.88_{-0.09}^{+0.07}$ & $-0.10_{-0.06}^{+0.05}$ & $-0.32_{-0.34}^{+0.35}$ & $0.25 \pm 0.13$ & $-1.88_{-0.61}^{+0.63}$ \\
\hline
\end{tabular}

Table 1: Results of fitting to the lattice data of the scattering lengths with 5 parameters.

\begin{tabular}{|l|ccccc|}
\hline Channels & $D \bar{K}(I=1)$ & $D \bar{K}(I=0)$ & $D_{s} K$ & $D \pi(I=3 / 2)$ & $D_{s} \pi$ \\
\hline$a(\mathrm{fm})$ & $-0.20(1)$ & $0.84(15)$ & $-0.18(1)$ & $-0.100(2)$ & $-0.002(1)$ \\
\hline
\end{tabular}

Table 2: The scattering lengths extrapolated to the physical light quark masses.

\begin{tabular}{|lcccc|}
\hline Channels & $D \pi(I=1 / 2)$ & $D K(I=0)$ & $D K(I=1)$ & $D_{s} \bar{K}$ \\
\hline$a(\mathrm{fm})$ & $0.37_{-0.02}^{+0.03}$ & $-0.84_{-0.22}^{+0.17}$ & $0.07 \pm 0.03+i\left(0.17_{-0.01}^{+0.02}\right)$ & $-0.09_{-0.05}^{+0.06}+i(0.44 \pm 0.05)$ \\
\hline
\end{tabular}

Table 3: Scattering lengths of $D \pi(I=1 / 2), D K(I=1)$ and $D_{s} K$ at the physical pion mass predicted from the fit.

Ref. [11]. There are six low-energy constants (LECs): $h_{0}, h_{1}, h_{24}, h_{4}^{\prime}, h_{35}$ and $h_{5}^{\prime}$. The value of $h_{1}$ is fixed to be 0.24 from the SU(3) mass splitting of the charmed mesons. There are still six parameters, which are $\tilde{a}, h_{0}, h_{24}, h_{4}^{\prime}, h_{35}$ and $h_{5}^{\prime}$, to be determined by fitting them to the lattice data.

For a pion mass as high as $617 \mathrm{MeV}$, the kaon mass would be even higher, around $700 \mathrm{MeV}$. Such values are too large for a controlled chiral expansion. Therefore, we will only fit to the ensembles $\mathrm{m} 007, \mathrm{~m} 010$ and $\mathrm{m} 020$. The best fit has $\chi^{2} /$ d.o.f $=1.06$, and the resulting parameters are collected in Table 1. At the physical pion mass, the extrapolated scattering lengths for the five channels are presented in Table 2.

Once we have determined the LECs in the chiral Lagrangian, we can make predictions on the scattering lengths of those channels we did not calculate due to the computational difficulties. The results for the scattering lengths of $D \pi(I=1 / 2), D K(I=0), D K(I=1)$ and $D_{s} \bar{K}$ at the physical pion mass are presented in Table 3.

The most interesting channel is the one with $(S, I)=(1,0)$, where the $D_{s 0}^{*}(2317)$ resides. The attraction in this channel is so strong that a pole emerges in the resummed amplitude. Within the range of $1 \sigma$ uncertainties of the the parameters, there is always a pole on the real axis in the first Riemann sheet, which corresponds to a bound state. If we use the physical values for all the meson masses, the pole position is $2315_{-28}^{+18} \mathrm{MeV}$. The central value corresponds to the pole found using the best fit parameters. It is very close to the observed mass of the $D_{s 0}^{*}(2317),(2317.8 \pm 0.6) \mathrm{MeV}$, and it is found in the channel with the same quantum numbers as that state. Therefore, one is encouraged to identify the bound state pole with the $D_{s 0}^{*}(2317)$.

As emphasized in, for instance, Refs. [14, 15], if there is an $S$-wave shallow bound state, the scattering length is related to the binding energy, and to the wave function renormalization constant $Z$, with $(1-Z)$ being the probability of finding the molecular component in the physical state (for $Z=0$, the physical state is purely a bound state). The relation reads

$$
a=-2\left(\frac{1-Z}{2-Z}\right) \frac{1}{\sqrt{2 \mu \varepsilon}}(1+\mathscr{O}(\sqrt{2 \mu \varepsilon} / \beta))
$$

where $\mu$ and $\varepsilon$ are the reduced mass and binding energy, respectively. Were the $D_{s 0}^{*}(2317)$ a pure $D K$ bound state $(Z=0)$, the value of $D K(I=0)$ scattering length would be $a=-1.05 \mathrm{fm}$, which 


\begin{tabular}{|cccccc|}
\hline Fitting range & $\chi^{2} /$ d.o.f & $h_{24}$ & $h_{4}^{\prime}$ & $h_{35}$ & $h_{5}^{\prime}$ \\
\hline $\mathrm{m} 007-\mathrm{m} 020$ & 0.97 & $-0.10_{-0.06}^{+0.05}$ & $-0.30_{-0.28}^{+0.31}$ & $0.26_{-0.10}^{+0.09}$ & $-1.94_{-0.38}^{+0.46}$ \\
\hline
\end{tabular}

Table 4: Results of fitting to the lattice data of the scattering lengths with 4 parameters. The subtraction constant is solved from fixing the pole in the $(S, I)=(1,0)$ channel to $2317.8 \mathrm{MeV}$.

\begin{tabular}{|l|ccccc|}
\hline Channels & $D \bar{K}(I=1)$ & $D \bar{K}(I=0)$ & $D_{s} K$ & $D \pi(I=3 / 2)$ & $D_{s} \pi$ \\
\hline$a(\mathrm{fm})$ & $-0.21(1)$ & $0.84(15)$ & $-0.18(1)$ & $-0.100(1)$ & $-0.002(1)$ \\
\hline
\end{tabular}

Table 5: The scattering lengths extrapolated to the physical light quark masses from the 4-parameter fit.

\begin{tabular}{|l|cccc|}
\hline Channels & $D \pi(I=1 / 2)$ & $D K(I=0)$ & $D K(I=1)$ & $D_{s} \bar{K}$ \\
\hline$a(\mathrm{fm})$ & $0.37 \pm 0.01$ & $-0.86 \pm 0.03$ & $0.04_{-0.01}^{+0.05}+i\left(0.16_{-0.01}^{+0.02}\right)$ & $-0.06_{-0.05}^{+0.01}+i(0.45 \pm 0.05)$ \\
\hline
\end{tabular}

Table 6: Scattering lengths of $D \pi(I=1 / 2), D K(I=0), D K(I=1)$ and $D_{s} K$ at the physical pion mass predicted from the 4-parameter fit.

coincides with the range in Table 3. From Eq. (3.2), the factor $Z$ is found to be in the range $[0.27,0.34]$. This means that the main component of the pole, corresponding to the $D_{s 0}^{*}(2317)$, is the $S$-wave $D K$ in the isoscalar channel.

\section{Isospin breaking width of the $D_{s 0}^{*}(2317)$}

In the following, we will assume that the $D_{s 0}^{*}(2317)$ corresponds to the pole generated in the $(S, I)=(1,0)$ channel, and explore the implications of our lattice calculation on this state. We will fix the pole position to the mass of the $D_{s 0}^{*}(2317), 2317.8 \mathrm{MeV}$, on the first Riemann sheet. We fit the lattice results of the scattering lengths with four parameters $h_{24}, h_{35}, h_{4}^{\prime}$ and $h_{5}^{\prime}$, and adjust the subtraction constant $\tilde{a}(\lambda=1 \mathrm{GeV})$ to reproduce the mass of the $D_{s 0}^{*}(2317)$. Again, we only fit to the ensembles $\mathrm{m} 007, \mathrm{~m} 010$ and $\mathrm{m} 020$. The best fit gives $\chi^{2} /$ d.o.f $=0.97$, which is slightly smaller than the one with one more parameter in Section 3. The parameter values together with the $1 \sigma$ statistical uncertainties are given in Table 4. At the physical pion mass, the extrapolated values of the scattering lengths are listed in Table 5. The results are quite similar to the ones in the last section, yet with slightly smaller uncertainties.

With the newly fitted parameters, the scattering lengths for several other channels are predicted, and the results are listed in Table 6. Again, the values are compatible with the ones in Table 3. Using Eq. (3.2), the value of $Z$ is again in the range of $[0.27,0.34]$. Both the stability of the fit and the small $Z$ indicates that the main component of the $D_{s 0}^{*}(2317)$ is the isoscalar $D K$ molecule.

We show the predictions for the pion mass dependence of the scattering lengths for the $D \pi(I=$ $1 / 2)$ and $D K(I=0)$ channels using parameters from this fit in Fig 2. Our prediction for the $D K(I=$ $0)$ channel is consistent with the very recent direct lattice calculation of this channel [16]. For the $D \pi(I=1 / 2)$ channel, our result at a pion mass of about $266 \mathrm{MeV}$ is larger than $(0.81 \pm 0.14) \mathrm{fm}$ obtained in [17]. From Fig. 2, one sees that such a pion mass is close to the transition point where the scattering length changes sign due to the generation of a pole (for more discussions, see [13]). In such a region, the value of the scattering length changes quickly. 

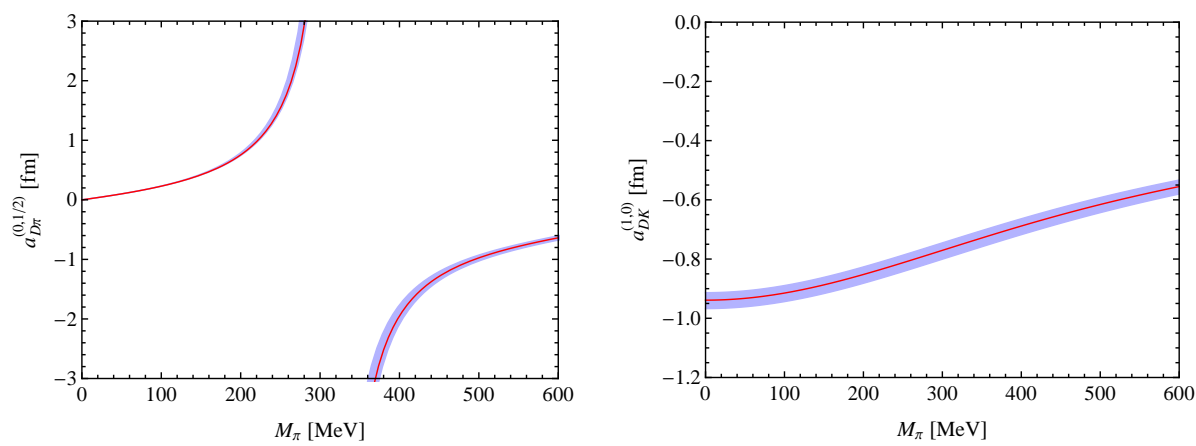

Figure 2: Predicted pion mass dependence of the $D \pi(I=1 / 2)$ and $D K(I=0)$ scattering lengths using parameters from the 4-parameter fit. The solid curves are calculated using the parameters from the best fit, and the bands reflect the uncertainties.

All the above calculations have assumed the same mass for the up and down quarks, and neglected the electromagnetic interaction. This is the isospin symmetric case. However, the $D_{s 0}^{*}(2317)$ decays into the isovector final state $D_{s} \pi$. In order to calculate this isospin breaking decay width, one has to take into account both the up and down quark mass difference and virtual photons. With the values of all the $h_{i}$ 's in Table 4, we calculate the isospin breaking decay width to be

$$
\Gamma\left(D_{s 0}^{*}(2317) \rightarrow D_{s} \pi\right)=(133 \pm 22) \mathrm{keV} .
$$

We have used the isospin breaking quark mass ratio $\left(m_{d}-m_{u}\right) /\left(m_{s}-\hat{m}\right)=0.0299 \pm 0.0018$, where $\hat{m}=\left(m_{u}+m_{d}\right) / 2$, which is calculated using the lattice averages (up to end of 2011) of the light quark masses. The error quoted in Eq. (4.1) comes from the uncertainties of the isospin breaking quark mass ratio, the $h_{i}$ 's and the chiral extrapolation, all added in quadrature.

\section{Summary}

The low-energy interaction between a light pseudoscalar meson and a charmed pseudoscalar meson was studied. We have calculated scattering lengths of five channels $D \bar{K}(I=0), D \bar{K}(I=$ $1), D_{s} K, D \pi(I=3 / 2)$ and $D_{s} \pi$ with four ensembles. The chiral extrapolation was performed using SU(3) unitarized chiral perturbation theory, and the LECs $h_{i}$ 's in the chiral Lagrangian were determined from a fit to the lattice results. With the same set of LECs and the masses of the involved mesons set to their physical values, we made predictions on other channels including $D K(I=0), D K(I=1), D \pi(I=1 / 2)$ and $D_{s} \bar{K}$. In particular, we found that the attractive interaction in the $D K(I=0)$ channel is strong enough so that a pole is generated in the unitarized scattering amplitude. Within $1 \sigma$ uncertainties of the parameters, the pole is at $2315_{-28}^{+18} \mathrm{MeV}$, and it is always below the $D K$ threshold. From calculating the wave function normalization constant, it is found that this pole is mainly an $S$-wave $D K$ bound state. By further fixing the pole to the observed mass of $D_{s 0}^{*}(2317)$, we revisited the isospin breaking decay width of the $D_{s 0}^{*}(2317) \rightarrow D_{s} \pi$. The result $(133 \pm 22) \mathrm{keV}$ updates the old result $(180 \pm 110) \mathrm{keV}$ obtained in Ref. [18]. It is nice to see that the uncertainty of the width shrinks a lot. We want to stress that the width is much larger than the isospin breaking width of a $c \bar{s}$ meson, which is of order $10 \mathrm{keV}$. 


\section{Acknowledgements}

We thank the NPLQCD and LHP Collaborations for sharing their light and strange propagators. We also thank André Walker-Loud and Huey-Wen Lin for important contributions to this work. Calculations were performed using the Chroma software suite, on computer clusters at Jefferson Laboratory (USQCD SciDAC supported) and the College of William and Mary (Cyclades cluster supported by the Jeffress Memorial Trust grant J-813). The work of L. L. and K. O. is supported in part by Jefferson Science Associates under U.S. DOE Contract No. DE-AC0506OR23177, and in part by DOE grants DE-FG02-07ER41527 and DE-FG02-04ER41302. L. L. also acknowledges support from the European Union under Grant Agreement number 238353 (ITN STRONGnet). The work of F.-K. G., C. H. and U.-G. M. is supported in part by the DFG and the NSFC through funds provided to the Sino-German CRC 110 "Symmetries and the Emergence of Structure in QCD", and the EU I3HP "Study of Strongly Interacting Matter" under the Seventh Framework Program of the EU. F.-K. G. also acknowledges partial support from the NSFC (Grant No. 11165005).

\section{References}

[1] BABAR Collaboration, B. Aubert et al., Phys.Rev.Lett. 90, 242001 (2003), [hep-ex/0304021].

[2] CLEO Collaboration, D. Besson et al., Phys.Rev. D68, 032002 (2003), [hep-ex/0305100].

[3] S. Godfrey and N. Isgur, Phys.Rev. D32, 189 (1985).

[4] C. W. Bernard et al., Phys.Rev. D64, 054506 (2001), [hep-lat/0104002].

[5] M. G. Alford, W. Dimm, G. P. Lepage, G. Hockney and P. B. Mackenzie, Phys. Lett. B361, 87 (1995), [hep-lat/9507010].

[6] MILC, K. Orginos, D. Toussaint and R. L. Sugar, Phys. Rev. D60, 054503 (1999), [hep-lat/9903032].

[7] D. B. Kaplan, Phys. Lett. B288, 342 (1992), [hep-lat/9206013].

[8] A. X. El-Khadra, A. S. Kronfeld and P. B. Mackenzie, Phys. Rev. D55, 3933 (1997), [hep-lat/9604004].

[9] M. Lüscher, Commun.Math.Phys. 105, 153 (1986).

[10] M. Lüscher, Nucl.Phys. B354, 531 (1991).

[11] L. Liu, K. Orginos, F.-K. Guo, C. Hanhart and U.-G. Meissner, Phys.Rev. D87, 014508 (2013), [1208.4535].

[12] S. Beane, P. Bedaque, A. Parreno and M. Savage, Phys.Lett. B585, 106 (2004), [hep-lat/0312004].

[13] F.-K. Guo, C. Hanhart and U.-G. Meißner, Eur.Phys.J. A40, 171 (2009), [0901.1597].

[14] S. Weinberg, Phys.Rev. 137, B672 (1965).

[15] V. Baru, J. Haidenbauer, C. Hanhart, Y. Kalashnikova and A. E. Kudryavtsev, Phys.Lett. B586, 53 (2004), [hep-ph/0308129].

[16] D. Mohler, C. Lang, L. Leskovec, S. Prelovsek and R. Woloshyn, 1308.3175.

[17] D. Mohler, S. Prelovsek and R. Woloshyn, Phys.Rev. D87, 034501 (2013), [1208.4059].

[18] F.-K. Guo, C. Hanhart, S. Krewald and U.-G. Meißner, Phys.Lett. B666, 251 (2008), [0806.3374]. 